\title{
EL PAPEL DE LA INFORMACIÓN FINANCIERA EN LA EVOLUCIÓN RECIENTE DEL SECTOR BANCARIO ESPAÑOL
}

\section{THE ROLE OF FINANCIAL INFORMATION ON RECENT DEVELOPMENTS IN SPANISH BANKING SECTOR}

\author{
Ruiz Rodríguez, María del Consuelo (Universidad de Jaén) * \\ Castilla Polo, Francisca (Universidad de Jaén) ${ }^{* * *}$ \\ RESUMEN
}

En este trabajo nos dedicamos a analizar el importante número de medidas adoptadas sobre el Sistema Financiero Español ante la crisis económica de los últimos años, así como a estudiar su repercusión en las estrategias contables de las entidades financieras, en un intento de adaptarse a tales cambios. Analizaremos las principales medidas financieras planteadas en los últimos años distinguiendo entre las encaminadas a aumentar la liquidez y las centradas en aumentar la solvencia de la entidad, todo ello con el propósito de conseguir ofrecer una visión agregada de los efectos de tales medidas en la información financiera que elaboran estas entidades, que nos permita deducir la oportunidad y conveniencia logradas por tal proceso de reforma.

Palabras claves: Entidades financieras, información financiera, confianza, transparencia. JEL: G21.

\begin{abstract}
This paper is dedicated to analyze the large number of measures taken on the Spanish financial system before the economic crisis of recent years and to study their impact on financial strategies of financial institutions, in an attempt to adapt to such changes. Analyze key financial measures referred to our financial system since the early beginnings of the crisis, distinguishing between measures to increase liquidity and regain the confidence of investors and measures focused on increasing the solvency and transparency of the entity and its accounting impact on the balance sheets of financial institutions, all with the proposal to achieving offer an aggregate view of the effects of such measures on bank accounting that allows us to deduce the opportunity and convenience achieved.
\end{abstract}

Key words: Financial institutions, financial, trust, transparency. JEL: G21.

\section{INTRODUCCIÓN}

Las entidades financieras, como intermediarios financieros de la economía, que canalizan el ahorro hacia la inversión entre los distintos grupos de interés, han asumido una gran relevancia en la economía global actual, no tanto por su función sino por la forma en que la misma se está desarrollando, con innumerables críticas y pronunciamientos desde todos los

* Investigadora Grupo de Investigación SEJ289. cheloruizrodriguez@ gmail.com

* Departamento de Economía Financiera y Contabilidad, Facultad de Ciencias Sociales y Jurídicas, Campus las Lagunillas, s/n, 23071, Jaén. fpolo@ujaen.es

Recibido: Noviembre de 2016. Aceptado: Diciembre de 2016. 
sectores y colectivos. En este contexto, debemos destacar el papel de las recientes medidas de saneamiento financiero planteadas tanto a nivel de la Unión Europea por la Nueva Autoridad Bancaria Europea como para el caso español dictadas por el Ministerio de la Presidencia y que avalan la necesidad de reformar y estructurar su funcionamiento. Este reciente proceso de reforma de las entidades financieras centra nuestro interés, planteándonos conocer por qué motivos se está cuestionando el funcionamiento de este tipo de entidades y cuáles son los requisitos informativos exigibles hacia las mismas en este nuevo contexto de crisis económica y problemas de confianza.

La explicación a la situación actual se encuentra en que después de la explosión de la burbuja, el sector privado ha estado reduciendo su deuda al mínimo. Esto es lo que se denomina una recesión de balances. En este tipo de recesiones, se intenta llevar a cero la tasa de interés. Así, para evitar el colapso de la actividad económica se utiliza la política fiscal, es decir, que el gobierno incremente el gasto financiándose con el ahorro que realiza el sector privado para reducir su déficit presupuestario (González et al., 2014).

Además, esta situación actual se ha visto influenciada por la internacionalización de la banca española. En opinión de Parejo (2007), el año decisivo de esta internacionalización en el que se modifican los patrones de crecimiento de la gran banca española fue 1996. El elevado coste de entrada en otros mercados europeos obliga a la gran banca española a adoptar medidas de crecimiento externo, amparadas en el establecimiento de alianzas y acuerdos de cooperación con socios de mercados no comunitarios. En consecuencia, la gran banca española impulsa notablemente la colocación de activos en Latinoamérica durante toda la década de los noventa. Ejemplo de ello podemos apreciarlo en Banco Santander y BBVA, los cuales a finales de 2006 aún seguían en proceso de internacionalización.

Sin embargo, en opinión de Ontiveros y Baliña (2012), existe cierto grado de vulnerabilidad en este proceso de internacionalización de España relacionado con el endeudamiento de los agentes privados y con la posición dominante que ha desempeñado el sistema bancario nacional. En este sentido, la reciente crisis financiera parece haber ralentizado los flujos de inversión de España en el extranjero durante dos fases claramente diferenciadas: la primera, de 2009 a 2010 y la segunda, que comenzó en 2013 y continúa en la actualidad, donde la desinversión, junto con el efecto valoración negativa, resultó en una desaceleración de la inversión externa en las dos últimas décadas (Baliña y Berges, 2014). En opinión de García et al. (2007), la actual crisis se puede considerar de liquidez motivada por la pérdida de confianza en el seno del sistema financiero, ante la incertidumbre sobre la magnitud de las potenciales pérdidas y la localización de las mismas.

De forma más concreta, en el presente trabajo nos encargamos de analizar el papel que la información contable desempeña a lo largo de la evolución reciente del sistema financiero, resultando muy interesante en un momento como el presente donde se hacen evidentes los problemas de confianza por parte de los inversores ante la información transmitida por las entidades financieras. Especialmente nos interesa conocer cómo estas medidas están afectando al conjunto de la información financiera que obligatoriamente estas entidades han de elaborar, enfocándonos en dos requisitos fundamentales en este tema: la transparencia y la confianza, y que mayoritariamente están siendo fomentadas por las recientes normativas que afectan a las entidades financieras. De hecho, la regulación puede ser considerada como una de las principales respuestas a la situación de crisis en la que nos encontramos, por el incremento de normativa enfocada a controlar las actividades bancarias que se está produciendo en los últimos años (Carbó, 2012).

La literatura contable ya ha planteado la necesidad de realizar modificaciones financieras que intenten aumentar los niveles de transparencia informativa de las empresas en 
general para así ganar credibilidad y confianza ante los inversores [Ferreira et al. (2012), Crumpton (2011), Damodaran (2007)]. Destacan los trabajos que relacionan la toma de decisiones de inversores y accionistas basada en su confianza hacia la organización [Doinea et al. (2011), Zolfalizadeh et al. (2011), Sharkie (2009), Alonso y Bueno (2008)] y aquellos que describen la influencia del tamaño, la rentabilidad y el endeudamiento en la consecución de transparencia informativa [Bosón et al. (2006), Gómez et al. (2005), Kan y Pang (2005)]. Por su parte, algunos autores como Crumpton (2011) y Klotz et al. (2008) miden el impacto de formalizar el proceso de actuación de los líderes en la transparencia de la organización, mientras que otros como Ferreira et al. (2012), Longinos et al. (2012) y Popescu (2010) apuestan por el cumplimiento de estándares y códigos de conducta para demostrar dicha transparencia.

Si bien, para el caso de las entidades financieras, lo que se plantea es analizar el proceso de elaboración de la información contable bajo la influencia de factores como la reducción de la propensión al riesgo y del protagonismo de la banca de inversión (Torrero, 2009) e instrumentos de capitalización y reforma de las entidades [Aríztegui (2010) y García (2003)]. Es más, ciertos autores como Corral et al. (2011) están de acuerdo en afirmar que la eficiencia y los requisitos de transparencia exigidos por las autoridades nacionales han pasado a ser requisitos para asegurar su propia supervivencia.

Podemos considerar el sistema financiero español como un sistema diversificado, moderno, competitivo y completamente integrado en los mercados financieros internacionales [Corral et al. (2011), Aríztegui (2010), Campa y García (2008) y Parejo (2007)], si bien, lo anterior no elimina el general cuestionamiento sobre su funcionamiento al que nos acabamos de referir. Nuestro estudio está centrado en el ámbito español y tiene por objeto hacer una revisión de las medidas de reestructuración propuestas para el mismo. Lo anterior nos permitirá estudiar cómo cada una de las normativas existentes se inclinan hacia cuestiones como sanear la información contable y financiera, aumentar la solvencia y liquidez de las entidades financieras, fortalecer sus decisiones de capital o prevenir el riesgo de los mercados financieros, en definitiva nos adentraremos en conocer las implicaciones contables de dos demandas repetidas para la información contable de estas entidades: que aumenten su transparencia y que podamos confiar en su actuación. Constituye ésta la principal aportación de nuestro estudio, que no sólo describe las diferentes normativas emitidas para este tipo de empresas, sino que analiza su utilidad contable en el sentido de favorecer las dos exigencias más repetidas hacia su gestión.

Para ello, en un segundo apartado abordamos la situación de las entidades financieras en el mercado financiero actual como contexto de estudio. A continuación, en el tercero, nos centraremos en la función de intermediación que llevan a cabo y, en especial, al papel de la confianza en su desarrollo. En el cuarto nos ocuparemos de analizar las diferentes normativas que han afectado al sector financiero y sus principales implicaciones para la información financiera como objetivo de investigación. Dedicaremos un quinto apartado a analizar las principales medidas adoptadas por el Banco Central Europeo ante esta crisis financiera y finalizaremos con el comentario de las conclusiones más relevantes y con el detalle de las principales limitaciones y futuras líneas de investigación.

\section{LAS ENTIDADES FINANCIERAS COMO OBJETIVO DE ESTUDIO}

La reciente internacionalización de las economías nos lleva a considerar la complejidad, interdependencia y flexibilidad del entorno económico, financiero y social en el que interactúan los distintos países, destacando la intensificación de las relaciones establecidas entre los mismos [Campa y García (2008), Dunning (1994), Ohmae (1991)]. En esta 
internacionalización, se ha producido una apertura de los mercados, marcada por la importancia de las relaciones financieras.

No obstante, los argumentos anteriores se encuentran claramente influenciados por la crisis financiera acaecida en todos los sectores de la economía [Mareque et al. (2011) y Rodríguez y Santos (2011)]. Algunos autores encuentran el origen de esta crisis económica en el sector de las entidades financieras, el cual repercute en el conjunto de las economías (Guillén y Tschoegl, 2008), siendo este argumento crucial para avalar el entramado de normativa reciente que sobre las mismas se ha desarrollado y que trataremos a continuación, así como las continuas críticas que, sobre su funcionamiento, se están realizando desde múltiples frentes.

No se encuentran atravesando una situación fácil, sino todo lo contrario, debemos destacar la etapa negativa que atraviesan las entidades financieras, debido al desarrollo de un conjunto de factores simultáneos. De hecho, suelen aparecer como protagonistas de la concreción de la ayuda financiera a la banca, en la regulación del sector, en el establecimiento de los fondos de rescate y parecen desempeñar un papel crucial en la confirmación del cambio de tendencia ocurrido en el comportamiento actual del sector. Igualmente, la difícil situación que apuntábamos para el sistema financiero español podemos observarla en los cambios significativos del número de entidades que conforman este sector.

Otra evidencia del cambio significativo en la estructura de este sector puede observarse a través del índice Herfinghal-Hirshman (HHI), entendido como la suma de los cuadrados de todas las cuotas de mercado. Este índice ha pasado de representar 821 puntos antes del proceso de integración a 1493 puntos, lo cual nos indica el aumento de concentración bancaria del sector (Carbó, 2012).

Problemas relacionados con la inmovilización general de los activos bancarios junto con un significativo incremento en la demanda por liquidez por parte de los depositantes, suelen ser apuntados como el origen de la situación bancaria actual. Destacan por otro lado los conflictos macroeconómicos (externos e internos) y microeconómicos, la implementación de programas de estabilización, programas de liberalización financiera y sistemas de supervisión y regulación deficientes [Campa y García (2008) y Guillén (2004)]. También consideramos importante subrayar la influencia negativa de otros aspectos del mercado interbancario, como la competencia de oferentes del mercado interbancario, el descenso de las cotizaciones medias de las entidades en los últimos años, la reducción de los ratios de eficiencia, rentabilidad financiera, económica, las elevadas tasas de morosidad y de cobertura de morosos, así como la reducción del número de oficinas y empleados en activo (Muñoz y Rodríguez, 2011).

Todas estas cuestiones que acabamos de apuntar repercuten, en última instancia, en la necesidad de mejorar la información de las entidades financieras, buscando la transparencia y coherencia de la misma con el objetivo de recuperar la confianza de inversores y público en general. Algunos autores como White (2012) consideran que el problema central que el sector financiero busca resolver es la reducción de la información asimétrica para que los ahorradores se sientan motivados a prestar su dinero y vuelva la liquidez y, por extensión, la senda de crecimiento a nuestra economía.

\section{LA INFORMACIÓN FINANCIERA DENTRO DE LA LABOR DE INTERMEDIACIÓN}

El sistema financiero se ocupa fundamentalmente de canalizar el ahorro hacia la inversión relacionando los diferentes grupos de interés, propiciando la inversión y dinamizando el proceso de crecimiento económico (figura 1). 


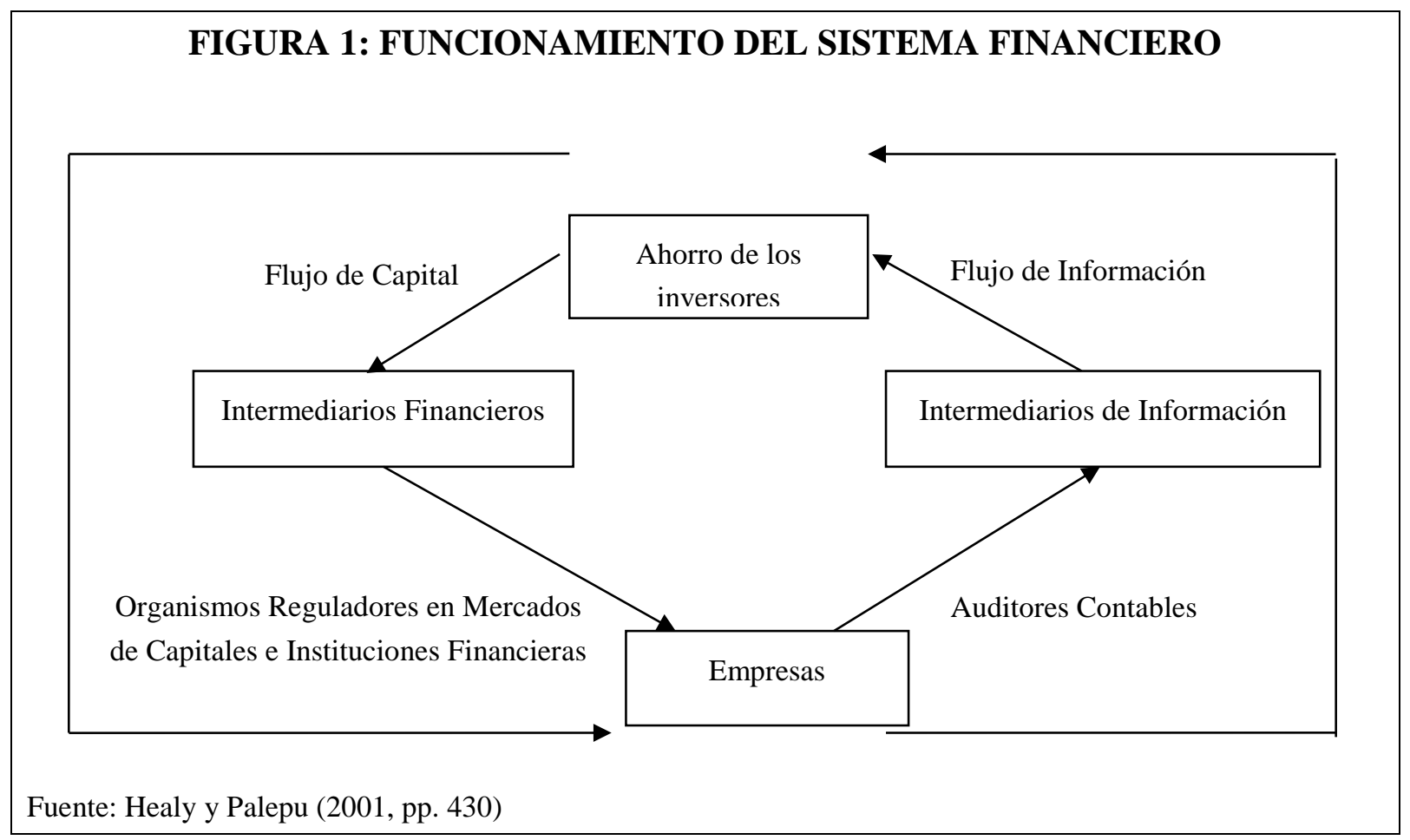

Analizando con mayor profundidad su actividad, podemos deducir que una de las funciones del sistema financiero consiste en distribuir el riesgo de la inversión con el objetivo de conseguir un crecimiento sostenido en la economía, mejorar la productividad y la eficiencia de las inversiones (Muñoz y Rodríguez, 2011). Así como otras relativas al mantenimiento del control de las tasas de interés, la emisión de dinero, bonos, divisas y otros títulos valor, dotando estabilidad monetaria y financiera a la economía [Pérez y Trucharte (2012), Corral et al. (2011), Aríztegui, (2010) y Parejo (2007)].

Nos centraremos especialmente en la función de intermediación de las entidades financieras donde aparecen ciertas debilidades como los auges crediticios, el diseño de la política económica, la diversificación del sector externo, el endeudamiento en moneda extranjera, el peso relativo del Estado en la propiedad de las entidades, la eficiencia de la supervisión, el desempeño financiero de los intermediarios bancarios y la distorsión de los incentivos y riesgo moral (Guillén y Tschoegl, 2008). Unidas las anteriores debilidades a una que consideramos fundamental, la pérdida de confianza de los grupos de interés. Es aquí donde la información financiera adquiere su papel fundamental, y así lo han entendido las diferentes autoridades regulatorias: Autoridad Bancaria Europea, Sistema Europeo de Bancos Centrales, Fondo Monetario Internacional, Comisión Nacional del Mercado de Valores y Banco de España, entre otros.

Resulta necesario disponer de información adecuada, sólida y transparente sobre la situación de la entidad financiera, lo cual podrá aumentar la calidad de la información para los inversores y recuperar la credibilidad de accionistas ante nuevas inversiones. De hecho, podemos afirmar que en la actualidad los gobiernos se encuentran en una disyuntiva por cuanto desean que las entidades financieras reduzcan su exposición al riesgo e incrementen su capital, pero por otro lado les piden que aumenten sus préstamos y restablezcan sus economías (White, 2012).

De este modo, consideramos que las medidas que recientemente han repercutido en los estados contables de las entidades financieras y que han sido diseñadas para atender a las 
demandas de crecimiento y estabilidad, también deberían de haberse planteado para atender las necesidades de los grupos de interés a los que desean satisfacer, permitiendo en última instancia el cumplimiento de la necesaria función de intermediación que este tipo de empresas llevan a cabo. La información asimétrica, como ya hemos comentado, es uno de los problemas que obstaculiza esta función y debería ser atendida por el aluvión de disposiciones y normativas que han surgido en los últimos años para estas entidades.

\subsection{El papel de la confianza dentro de la función de intermediación}

La función de intermediación del sistema financiero parte de un requisito fundamental, esto es, la confianza de los inversores sobre las diferentes entidades bancarias. En este sentido, debemos precisar qué se entiende por confianza en un sistema financiero.

Algunos autores como Delgado et al. (2012) y Rousseau et al. (1998) entienden la confianza como un estado psicológico que comprende la intención de aceptar la vulnerabilidad basada en expectativas positivas de las intenciones o comportamientos de otra persona. De ahí, que exista una relación de confianza interpersonal que involucra a dos personas: la persona que confía y la persona que ofrece la confianza (Delgado et al., 2012). Existe una importante bibliografía relacionada con la confianza y centrada en destacar las características específicas de las relaciones entre las personas ante el supuesto de que la confianza implica expectativas favorables sobre las acciones de otras personas [Delgado et al. (2012), Hardin (2002), Putnam (1995), Coleman (1990), Gambetta (1988), Luhmann (1979)]. Otra nota distintiva importante empleada en la definición de este concepto es el grado en el que una de las partes está dispuesta a depender de alguien, o algo, en una situación dada con una sensación de relativa seguridad, aunque las consecuencias negativas sean posibles (Josang y Presti, 2004). Para algunos autores como Clarkson et al. (1986) la confianza es el deber de actuar en beneficio de otra persona manteniendo una relación de certidumbre y tranquilidad, e implica ciertos deberes como el rendimiento, la notificación, la legalidad y la obediencia.

Si aplicamos el concepto confianza al ámbito financiero podemos indicar que este concepto se refiere a la estabilidad general del sistema financiero y de los ciudadanos en sus instituciones. Es lo que se denomina confianza sistémica en la política e instituciones a nivel europeo y nacional y en la economía de libre mercado (Roth, 2011).

Sin lugar a dudas, el papel de la información es fundamental en el establecimiento de una relación de confianza, tanto a nivel general, como a nivel particular para las relaciones de intermediación financiera. Es precisamente este objetivo, el papel desarrollado por la información financiera en la mejora de la confianza, atendido a través de las recientes medidas de reforma que afectan al sector de las entidades financieras, el que vamos a analizar en este trabajo. De ahí, la trascendencia de analizar la evolución experimentada por los indicadores que tratan de acercarse a la cuantificación de este concepto, cuestión que pasamos a revisar sin pretender centrar en ello el objetivo de nuestro estudio pero sí evidenciando su papel subyacente en el mismo.

La confianza se puede aproximar a la medida del riesgo soberano del país debido a que se trata de un indicador que mide las condiciones económicas, sociales, políticas o, incluso, naturales y geográficas, que generan un nivel de riesgo específico para las inversiones, evaluado de acuerdo al conocimiento y opinión de los inversores. Los elevados niveles de prima que sufrió nuestro país parecen estar controlándose y a finales de 2013 la prima se ha situado en 220 puntos. Otra medida descriptiva de la confianza relativa al sistema financiero nos la proporciona el Índice de Confianza del Consumidor que nos permite aproximarnos a 
las intenciones de gasto de los consumidores preguntándoles por su percepción actual y sus expectativas de futuro para la economía del país, su economía familiar y el empleo, indicador que estaba situado en 71 puntos en diciembre de 2013.

Otro indicador empleado en el análisis de la confianza es el indicador de estabilidad financiera que proporciona una instantánea de las condiciones de crédito y del movimiento conjunto de la tensión entre las economías avanzadas y emergentes. Además, capta la tensión financiera y los niveles de estrés de las economías, los cuales originan pérdida de confianza. Así, este índice alcanza su máximo en 2008 rondando los 8 puntos, descendiendo en 2009 hasta casi los 5 puntos y en el período 2012-2013, vuelve a marcar una tendencia decreciente situándose en los 2 puntos.

Finalmente, otros instrumentos de medida procedentes del ámbito privado para el tema de la confianza son el barómetro "Edelman Trust Barometer" y el estudio "European Social Survey". El primero mide el estado de confianza del público en general analizando la confianza en las entidades financieras de un modo específico y el impacto de la reciente crisis en el sector bancario. Este índice ha aumentado para los bancos y muestra un nivel de confianza del 43 por 100 en 2012 frente al 51 por 100 en 2016. Por su parte, el European Social Survey incluye entre uno de sus temas, la confianza y su aplicación a los medios de comunicación y al ámbito social. Este indicador utilizado ampliamente dentro de la investigación sociológica incluye referencias específicas según país aunque con carácter menos específico para al ámbito de las instituciones financieras.

\section{IMPLICACIONES FINANCIERAS DE LAS NUEVAS MEDIDAS DE REESTRUCTURACIÓN PROPUESTAS PARA EL SECTOR FINANCIERO}

A nivel europeo se están produciendo un conjunto de reformas financieras con el objetivo de fortalecer las dotaciones de capital de las entidades bancarias y prevenir el riesgo sistémico en los mercados financieros lideradas por la figura de la Autoridad Bancaria Europea. Podemos decir que a consecuencia de la crisis se están produciendo numerosas reformas en los mercados financieros europeos, tales como el Nuevo Acuerdo de Basilea III sobre requerimientos de capital para la banca ${ }^{1}$ para asegurar la estabilidad de las instituciones financieras. El nuevo marco que establece Basilea III supone una exigencia notable para las entidades en términos de más y mejor capital regulador, cobertura de la liquidez y lucha contra la expansión y contracción del crédito asociada al ciclo económico a través de las nuevas disponibilidades de capital. Incluso, en este acuerdo se establecen unas exigencias de capital mayores para las cajas de ahorros, lo cual puede interpretarse cono un mecanismo indirecto para convertirse en bancos, en la medida en que sólo así podrán apelar al nuevo capital exigido, sea en mercados, o sea a través del nuevo Fondo de Reestructuración Ordenada Bancaria (FROB), que sólo se podrá instrumentar mediante entrada en el capital (Berges y Martín, 2011). Además, según el informe del Centro del Sector Financiero de PwC y el Business School $(2010)^{2}$ se exige un capital de mayor calidad, con mayor liquidez, menor apalancamiento y mayor solvencia que favorecerá la estabilidad macroeconómica.

\footnotetext{
1 El Nuevo Acuerdo de Basilea III está compuesto por dos documentos: uno sobre medidas referidas al capital y a los ratios de solvencia y otro de medidas relativas a la liquidez, el cual se implantó el 1 de enero de 2013 para cumplir con la fecha límite de aplicación de Basilea III. Información disponible http://www.eba.europa.eu. Acceso noviembre 2016.

2 La propuesta de Reglamento sobre Requisitos de Capital establece aspectos relativos a la administración de las entidades financieras sobre la amortización de máximos, definiciones de capital, tratamiento de exposiciones específicas, riesgo de crédito, liquidez y ratio de apalancamiento. Información disponible http://www.eba.europa.eu. Acceso noviembre 2016.
} 
Otras de las reformas es el Reglamento sobre Requisitos de Capital, los cuales se debían haber implantado en el año 2013, junto con la nueva normativa del ratio de cobertura de liquidez (Liquidity Coverage Ratio: LCR) para garantizar a corto plazo la resistencia de las instituciones financieras y recuperar la confianza de los inversores.

No obstante, para garantizar el cumplimiento de todas estas medidas nace la figura de la Autoridad Bancaria Europea (EBA), la cual tiene la obligación de proporcionar a los supervisores e instituciones europeas criterios, normas y asesoramiento técnico. Entre sus objetivos generales encontramos el de mantener la estabilidad financiera en la UE y velar por la integridad, la eficiencia y el correcto funcionamiento del sector bancario.

La repercusión de estas medidas a nivel español se ha traducido en numerosas modificaciones del sistema financiero. Así, para hacer frente a este período de recesión se han tomado medidas como los aumentos en la supervisión y de las exigencias de recursos propios para estas entidades (Real Decreto-Ley 11/2010, de 9 de julio). Igualmente, se han establecido requerimientos de cobertura adicionales a las entidades financieras para cubrir el deterioro de las financiaciones vinculadas a la actividad inmobiliaria y la creación de una sociedad de gestión de activos relacionada con las promociones inmobiliarias (Real Decreto-Ley 18/2012, de 12 de mayo).

No obstante, todo el proceso de reestructuración del sistema financiero español, se puede remontar al año 2008 cuando se planteó el Fondo para la Adquisición de Activos Financieros (FAAF) para aumentar la liquidez mediante la publicación del Real Decreto-Ley 6/2008, de 10 de octubre. Debemos apuntar que España dispone del sistema de Fondos de Garantía de Depósitos (FGD), con el objetivo de garantizar los depósitos en dinero y en valores constituidos en las entidades de crédito, destacando tres apartados:

- Fondo de Garantía de Depósitos de Establecimientos Bancarios (FGDEB).

- Fondo de Garantía de Depósitos en Cajas de Ahorros (FGDCA).

- Fondo de Garantía de Depósitos en Cooperativas de Crédito (FGDCC).

Así, el proceso de reordenación bancaria parece realizarse a través de los Fondos de Garantía de Depósitos. De este modo, se pretende adoptar una serie de medidas con el objetivo de incrementar la fortaleza y solvencia del sistema bancario español. Un año más tarde, en 2009, se publicó el Real Decreto-Ley 9/2009 por el que se creaba el Fondo de Reestructuración Ordenada Bancaria con el fin de fortalecer la intervención y disciplina del sistema financiero español ${ }^{3}$. En 2010 las reformas se centraron en las Cajas de Ahorros, mediante el Real Decreto-Ley 11/2010, de 9 de julio de Órganos de Gobierno y otros aspectos del régimen jurídico de las Cajas de Ahorros, con el que se buscaba el acceso de las Cajas de Ahorros al mercado de capital básico.

Le siguen un importante número de medidas para fortalecer a las entidades financieras y elevar su solvencia aumentando los requisitos mínimos de capital, mediante el Real DecretoLey 2/2011, de 18 de febrero, para el Reforzamiento del Sistema Financiero y el Real Decreto-Ley 2/2012, de 3 de febrero para el Reforzamiento del Sistema Financiero y la Solvencia de las Entidades Financieras, que plantea una nueva valoración de los activos tendentes a sanear los balances y aumentar la credibilidad y confianza del sistema financiero

\footnotetext{
${ }^{3}$ Se entiende por solvencia a la capacidad financiera (capacidad de pago) de la empresa para cumplir sus obligaciones de vencimiento a corto plazo y los recursos con que cuenta para hacer frente a tales obligaciones, o sea una relación entre lo que una empresa tiene y lo que debe. No debemos confundir solvencia con liquidez, ya que la liquidez consiste en tener el efectivo necesario que nos permita hacer el pago de los compromisos contraídos, mientras que solvencia sería contar con bienes y recursos suficientes para respaldar los deudas contraídas. Información disponible en http://www.bde.es. Acceso marzo 2016.
} 
español. Además, sobre la base de las necesidades de capital de las entidades financieras estimadas en los últimos meses, la nueva legislación propone la siguiente clasificación que recogemos en el cuadro 1 .

\begin{tabular}{|c|c|c|c|c|c|}
\hline \multicolumn{6}{|c|}{$\begin{array}{c}\text { CUADRO 1: CLASIFICACIÓN DE LAS ENTIDADES FINANCIERAS } \\
\text { EN FUNCIÓN DE SUS NECESIDADES DE CAPITAL }\end{array}$} \\
\hline Denominación & Composición & Actuación & $\begin{array}{l}\text { Cotización en } \\
\text { bolsa }\end{array}$ & $\begin{array}{l}\text { Premisa para } \\
\text { cotizar }\end{array}$ & $\begin{array}{l}\text { Plazo de presentación } \\
\text { de cada actuación }\end{array}$ \\
\hline Grupo 0 & $\begin{array}{l}\text { Entidades que no } \\
\text { tienen déficit de } \\
\text { capital }\end{array}$ & Nula & No & Nula & Nulo \\
\hline \multirow[b]{2}{*}{ Grupo 1} & \multirow{2}{*}{$\begin{array}{c}\text { Entidades } \\
\text { financieras } \\
\text { nacionalizadas con } \\
\text { participación de } \\
\text { capital del FROB }\end{array}$} & Reestructuración & \multirow[b]{2}{*}{ Sí } & Ayuda estatal & Octubre 2012 \\
\hline & & Liquidación y venta & & $\begin{array}{l}\text { Traspaso de activos } \\
\text { deteriorados a la } \\
\text { sociedad gestora de } \\
\text { activos }\end{array}$ & Diciembre 2012 \\
\hline \multirow[b]{2}{*}{ Grupo 2} & \multirow{2}{*}{$\begin{array}{l}\text { Entidades } \\
\text { financieras con } \\
\text { déficit de capital } \\
(\text { pruebas de } \\
\left.\text { resistencia }{ }^{4}\right) \text { con } \\
\text { ayuda estatal para } \\
\text { cubrir estos déficits }\end{array}$} & Reestructuración & \multirow[b]{2}{*}{ Sí } & Ayuda estatal & Octubre 2012 \\
\hline & & Liquidación y venta & & $\begin{array}{l}\text { Traspaso de activos } \\
\text { a sociedad gestora } \\
\text { de activos }\end{array}$ & Diciembre 2012 \\
\hline \multirow{3}{*}{ Grupo 3} & \multirow{3}{*}{$\begin{array}{c}\text { Entidades } \\
\text { financieras con } \\
\text { déficit de capital } \\
\text { (pruebas de } \\
\text { resistencia) con } \\
\text { aumentos de capital } \\
\text { privados }\end{array}$} & Recapitalización & \multirow{3}{*}{ No } & \multirow{3}{*}{ Nula } & Octubre 2012 \\
\hline & & $\begin{array}{l}\text { Inyección de bonos } \\
\text { convertibles en } \\
\text { acciones (necesidades } \\
\text { de capital }>2 \% \text { ) }\end{array}$ & & & Diciembre 2012 \\
\hline & & $\begin{array}{l}\text { Traspaso de activos a } \\
\text { sociedad gestora de } \\
\text { activos (resto de } \\
\text { entidades que no } \\
\text { reciben capital) }\end{array}$ & & & Junio 2013 \\
\hline
\end{tabular}

Tal y como podemos observar, las entidades financieras pueden desempeñar distintos tipos de actuaciones, en función de la categoría en la que estén encuadradas. Además, por este motivo apreciamos diferencias en las exigencias de información financiera en función del tipo de entidad, ya que a las entidades del grupo 3 se les exige requisitos informativos adicionales frente a las entidades de niveles inferiores.

El grupo 0, entidades que no tienen necesidades de capital, no deberá desarrollar ningún tipo de actuación adicional en materia informativa, lo que se traduce en que no existirán para

\footnotetext{
${ }^{4}$ El objetivo general de las pruebas de resistencia consistió en evaluar la resistencia del sistema bancario europeo en su conjunto, y la capacidad de los bancos para absorber posibles shocks relacionados con sus riesgos de crédito y de mercado, incluido el riesgo derivado de las inversiones en deuda soberana". Información disponible en http://www.bde.es. Acceso noviembre 2016.
} 
las mismas problemas adicionales de los ya presentes en cuanto a la confianza de los inversores como por ejemplo BBVA, Banco Santander o CaixaBank. Por su parte, el grupo 1, entidades con participación del Fondo de Reestructuración Ordenada Bancaria, como Bankia o Catalunya Caixa y el grupo 2, entidades que necesitan apoyo público en su capital, como Liberbank, Caja 3 o Banco Mare Nostrum, deberían aumentar la transparencia de sus respectivas informaciones financieras añadiendo planes de recapitalización y liquidando y vendiendo los activos que no puedan sanearse con el plan de recapitalización. En el último grupo, se incluyen las entidades financieras que pueden aumentar su capital con medios privados con el objetivo de conseguir la confianza de los inversores como Banco Popular, Banco Sabadell o Unicaja. En este caso, presentarán planes de recapitalización o inyectarán bonos convertibles en acciones si las necesidades de capital son inferiores al 2 por 100. En caso contrario, si no reciben capital, podría ser necesario llevar a cabo medidas adicionales como el traspaso de los activos a la sociedad gestora de activos, para conseguir liquidez, recuperar la confianza de los inversores, aumentar la solvencia de las entidades financieras y obtener transparencia en el mercado. Por tanto, nos encontramos ante el grupo que presenta más necesidades de justificar su actuación, cuestión que debe ser abordada, entre otros frentes, con su información financiera.

De manera general, la reestructuración del Sistema Financiero Español se puede resumir tal y como aparece en la figura 2 :

\section{FIGURA 2: REESTRUCTURACIÓN DEL SFE}

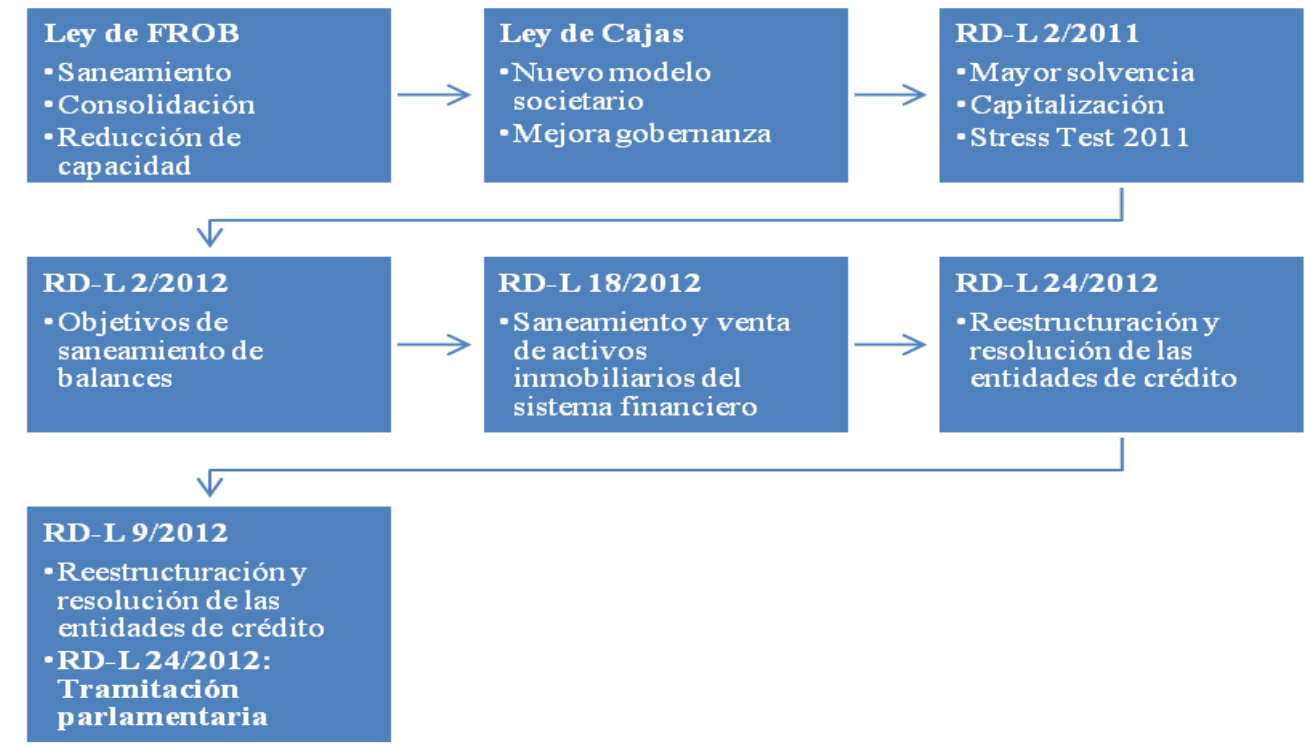

Fuente: Elaboración propia

Especialmente nos interesa identificar los efectos de las medidas adoptadas por el sistema financiero sobre las exigencias de transparencia que les exigen las repetidas críticas sobre su funcionamiento por parte de diversos colectivos y del público en general. También nos preocupa su efecto sobre la confianza, conseguida en parte a través del aumento de la cantidad de información revelada al exterior pero igualmente a través de las medidas de reestructuración propuestas para conseguir solvencia, así como los planes de recapitalización 
y el aumento de provisiones, los cuales se orientan a obtener liquidez, credibilidad y confianza entre accionistas e inversores.

\subsection{Actuaciones destinadas fundamentalmente a restablecer la liquidez de las entidades financieras}

Entre las primeras medidas desarrolladas para restablecer la liquidez de estas entidades, se incluirían fundamentalmente el Real Decreto-Ley 9/2009, el Real Decreto-Ley 11/2010 y el Real Decreto-Ley 2/2012, las cuales permiten un aumento de la transparencia informativa que reduzca la información asimétrica existente entre ahorradores y prestamistas y que, en última instancia, tenga sus efectos sobre la recuperación de los flujos de crédito bancario.

Los problemas de viabilidad y riesgo de la situación actual del sistema financiero justifican la necesidad de implantar una estrategia que favorezca la solución de los problemas mediante una reestructuración ordenada del sistema bancario español, con el objetivo de mantener la confianza en el sistema financiero nacional y de incrementar su fortaleza y solvencia de manera que las entidades que subsistan sean sólidas y puedan proveer crédito con normalidad. Sólo con esta información por parte de ahorradores e inversores se restablecerá la liquidez que necesitan estas entidades.

El Real Decreto-Ley 9/2009, de 26 de junio, sobre reestructuración bancaria y fortalecimiento de los recursos propios de las entidades de crédito, permite la creación del Fondo de Reestructuración Ordenada Bancaria con el fin de fortalecer la intervención, disciplina y solvencia del sistema financiero español. Entre las principales actuaciones planteadas por Real Decreto-Ley 9/2009 se incluirían las que se recogen en el cuadro 2. En concreto, se plantea la creación del FROB y la obtención de financiación con garantía del Estado. Además, se plantea el establecimiento de un plan de reestructuración con el objetivo de conseguir la viabilidad de la entidad.

Centrándonos en el caso de las cajas de ahorros, podemos decir que la crisis ha puesto de manifiesto la necesidad de una mayor flexibilidad para captar recursos básicos de capital y para ajustar sus estructuras operativas, aspectos sobre los que el Real Decreto-Ley 11/2010 ha configurado un conjunto de medidas que recogemos y analizamos en el cuadro 3. El Real Decreto-Ley 11/2010, de 9 de julio, de órganos de gobierno y otros aspectos del régimen jurídico de las cajas de ahorros, se dirige a fortalecer la captación de recursos de las cajas de ahorros españolas y su acceso al mercado de capitales, con el objetivo de aumentar su eficiencia y recuperar la confianza de los inversores. De forma más concreta, debemos comentar las exigencias de liquidez y de un límite de apalancamiento aplicables en función de la capacidad de la entidad para obtener recursos básicos (véase cuadro 3). 
CUADRO 2: MEDIDAS ADOPTADAS EN EL REAL DECRETO-LEY 9/2009

\begin{tabular}{|c|c|c|}
\hline Objetivos de intervención & Descripción de las actuaciones & Principales resultados perseguidos \\
\hline $\begin{array}{c}\text { Creación del Fondo de } \\
\text { Reestructuración } \\
\text { Ordenada Bancaria }\end{array}$ & $\begin{array}{l}\text { Igual régimen jurídico que los fondos de } \\
\text { garantía de depósitos. Financiación: } \\
\text { dotación de } 9.000 \text { millones de euros y } \\
\text { dotación de crédito para el Fondo para la } \\
\text { Adquisición de Activos Financieros }\end{array}$ & $\begin{array}{l}\text { La aparición del Fondo de } \\
\text { Reestructuración Ordenada Bancaria } \\
\text { posibilita la creación nuevas provisiones } \\
\text { que permiten elevar los niveles de } \\
\text { solvencia de las entidades financieras. Se } \\
\text { captará financiación ajena con garantía del } \\
\text { Estado. }\end{array}$ \\
\hline $\begin{array}{l}\text { Reestructuración de las } \\
\text { entidades de crédito }\end{array}$ & $\begin{array}{l}\text { Fases: plan de reestructuración para } \\
\text { reforzar el patrimonio y la solvencia de la } \\
\text { entidad, su fusión o absorción o el } \\
\text { traspaso total o parcial del negocio } \\
\text { mediante la cesión de activos y pasivos }\end{array}$ & $\begin{array}{l}\text { Se advierte la búsqueda de una solución } \\
\text { privada y la adopción de medidas de } \\
\text { viabilidad de las entidades de crédito para } \\
\text { aumentar la liquidez y la solvencia, } \\
\text { recibiendo apoyo financiero y de gestión. }\end{array}$ \\
\hline $\begin{array}{c}\text { Intervención del Fondo } \\
\text { de Reestructuración } \\
\text { Ordenada Bancaria }\end{array}$ & $\begin{array}{l}\text { Reforzar los recursos propios mediante la } \\
\text { adquisición de títulos: participaciones } \\
\text { preferentes convertibles en acciones, en } \\
\text { cuotas participativas o en aportaciones al } \\
\text { capital social. } \\
\text { Desembolso sin recompensar tras cinco } \\
\text { años implica su conversión en acciones, en } \\
\text { cuotas participativas o en aportaciones } \\
\text { sociales del emisor. }\end{array}$ & $\begin{array}{l}\text { Se refiere a la necesidad de aumentar la } \\
\text { liquidez de los balances de estas entidades } \\
\text { mediante previsiones de tesorería y } \\
\text { optimización del cash flow económico y } \\
\text { financiero. Las entidades financieras } \\
\text { podrán adquirir los activos con riesgo } \\
\text { elevado con el objetivo de inyectar } \\
\text { liquidez adicional en el mercado. }\end{array}$ \\
\hline
\end{tabular}

Fuente: Elaboración propia

\section{CUADRO 3: MEDIDAS ADOPTADAS EN EL REAL DECRETO-LEY 11/2010}

\begin{tabular}{|c|c|c|}
\hline Objetivos de intervención & Descripción de las actuaciones & Principales resultados perseguidos \\
\hline Cuotas participativas & $\begin{array}{l}\text { Consideración de recursos propios, } \\
\text { emisión de cuotas proporcionales al } \\
\text { patrimonio de caja, con límite del } 50 \% \text { del } \\
\text { patrimonio y cotización en mercados } \\
\text { secundarios. Fusión: canje por cuotas de la } \\
\text { entidad resultante de la fusión y } \\
\text { retribución, sin autorización } \\
\text { administrativa, salvo la del Banco de } \\
\text { España. }\end{array}$ & $\begin{array}{l}\text { Cada entidad utilizará las cuotas } \\
\text { participativas de un modo diferente. Así, el } \\
\text { canje de acciones efectuada la fusión y la } \\
\text { libertad de acción podrá aumentar la } \\
\text { solvencia y liquidez de la entidad. }\end{array}$ \\
\hline $\begin{array}{l}\text { Emisión, adquisición y } \\
\text { provisión de autocartera }\end{array}$ & $\begin{array}{l}\text { Libertad de emisión de cuotas y } \\
\text { cumplimiento del límite del } 5 \% \text { de } \\
\text { adquisición derivativa de sus propias } \\
\text { cuotas provisión a la autocartera de cuotas } \\
\text { para entidades centrales de un Sistema } \\
\text { Institucional de Protección. }\end{array}$ & $\begin{array}{l}\text { Se refiere al saneamiento de la autocartera, } \\
\text { mediante la libertad para emitir nuevas } \\
\text { provisiones y la aportación de transparencia. }\end{array}$ \\
\hline $\begin{array}{c}\text { Sistemas Institucionales } \\
\text { de Protección }\end{array}$ & $\begin{array}{l}\text { La entidad central habrá de estar } \\
\text { participada en al menos un } 50 \% \text { de su } \\
\text { accionariado y tener naturaleza de } \\
\text { sociedad anónima. }\end{array}$ & $\begin{array}{l}\text { Se advierte la importancia de un sistema de } \\
\text { protección para los inversores que } \\
\text { proporcionen confianza y seguridad en sus } \\
\text { actuaciones. }\end{array}$ \\
\hline Liquidez & $\begin{array}{l}\text { Nuevas exigencias de liquidez y un límite } \\
\text { al apalancamiento, aplicables en función } \\
\text { de la capacidad de la entidad para obtener } \\
\text { recursos propios básicos. }\end{array}$ & $\begin{array}{l}\text { Se advierte la posibilidad de hacer frente a } \\
\text { las salidas netas de efectivo que pudieran } \\
\text { producirse en escenarios adversos al } \\
\text { considerar activos plenamente disponibles y } \\
\text { obtener fondos con alto grado de liquidez. }\end{array}$ \\
\hline
\end{tabular}

Fuente: Elaboración propia 
Finalmente, debemos indicar que el Real Decreto-Ley 2/2012, ha sido clave en el esfuerzo orientado a recuperar la confianza y credibilidad de los inversores y sanear los activos problemáticos, mediante el establecimiento de nuevos planteamientos de recapitalización de las entidades financieras y de nuevos objetivos de liquidez -cuadro 4-. Los principales objetivos marcados en esta normativa, podemos resumirlos en medidas relativas al saneamiento del sector financiero, reestructuración bancaria y refuerzo de los recursos propios de las entidades de crédito (modificación del Real Decreto-Ley 9/2009, de 26 de junio), modificación de los órganos de Gobierno y otros aspectos del régimen jurídico de las Cajas de Ahorros (Real Decreto-Ley 11/2012, de 9 de julio) e introducción de un tratamiento especial para las entidades de crédito que tengan en circulación participaciones preferentes o deudas convertibles obligatoriamente (véase cuadro 4).

\begin{tabular}{|c|c|c|c|c|c|}
\hline \multicolumn{6}{|c|}{ CUADRO 4: MEDIDAS PROPUESTAS POR REAL DECRETO-LEY 2/2012 } \\
\hline $\begin{array}{c}\text { Cobertura } \\
\text { activos } \\
\text { inmobiliarios en } \\
\text { pago de deudas } \\
\text { de entidades } \\
\text { financieras }\end{array}$ & $\begin{array}{l}\text { Cobertura } \\
\text { operaciones } \\
\text { dudosas }\end{array}$ & $\begin{array}{l}\text { Operaciones } \\
\text { subestándar }\end{array}$ & $\begin{array}{l}\text { Temporalidad } \\
\text { máxima }\end{array}$ & $\begin{array}{l}\text { Plan de } \\
\text { reestructuración } \\
\text { y FROB }\end{array}$ & Ayuda \\
\hline $\begin{array}{l}40 \% \text { general } \\
\text { Construcciones. } \\
\text { Terminadas: } \\
30 \% \\
\text { Construcciones } \\
\text { en Curso: } 50- \\
60 \%\end{array}$ & $\begin{array}{l}25 \% \text { general } \\
\text { Suelo: } 60 \% \\
\text { Const.: } 50 \%\end{array}$ & $\begin{array}{c}\text { 20-24\% general } \\
\text { Suelo: } 60 \% \\
\text { Construcciones. } \\
\text { Terminadas: } \\
50 \% \\
\text { Construcciones } \\
\text { en Curso: } 24 \%\end{array}$ & $31 / 12 / 2012$ & $\begin{array}{c}\text { Sí } \\
\text { No apoyo } \\
\text { FROB }\end{array}$ & $\begin{array}{c}\text { Activos } \\
\text { convertibles en } \\
\text { acciones a } \\
\text { interés 7\% }\end{array}$ \\
\hline
\end{tabular}

Específicamente establece distintos tipos de coberturas para aportar liquidez como las destinadas al pago de deudas de entidades financieras, de operaciones dudosas o subestándar ${ }^{5}$, las cuales apenas son recogidas en el resto de medidas de saneamiento propuestas. Así, mediante estas nuevas coberturas, la construcción de un plan de reestructuración y las ayudas procedentes de activos convertibles en acciones, se ha intentado recuperar la confianza de inversores y accionistas, aportando liquidez al sistema y a cada una de las entidades financieras que lo conforman.

\subsection{Actuaciones destinadas fundamentalmente a reforzar la solvencia de las entidades financieras}

Junto a las anteriores medidas, destacamos la existencia de diversas actuaciones centradas mayoritariamente en aumentar la solvencia de las entidades financieras, y en divulgar tales cambios para mejorar la confianza en su gestión y restablecer el crecimiento

\footnotetext{
${ }^{5}$ Las operaciones subestándar son aquellas construcciones o promociones inmobiliarias de todo tipo de activos que se encuentran terminadas.
} 
económico. En concreto, estas medidas aparecen recogidas fundamentalmente por el Real Decreto-Ley 18/2012 y por el Real Decreto-Ley 24/2012.

El Real Decreto-Ley 18/2012, de 11 de mayo, sobre Saneamiento y Venta de los Activos Inmobiliarios del Sector Financiero, establece con el objetivo de lograr el saneamiento de los balances de las entidades de crédito afectados negativamente por el deterioro de los activos vinculados al sector inmobiliario, así como la disminución de las incertidumbres sobre el sector financiero español, la recuperación de la confianza de los mercados y la canalización del crédito a la economía real.

Además, las medidas adoptadas en el Real Decreto-Ley 18/2012, se dirigen especialmente a recuperar la solvencia. Establece coberturas adicionales a las entidades de crédito que oscilan desde, el 45 por 100 para el crédito promotor sin garantía hipotecaria o con suelo como garantía hipotecaria, el 22 por 100 como garantía de promociones en curso y el 7 por 100 como garantía de promociones terminadas. De esta forma, se advierte la relevancia de dotar nuevas provisiones para hacer frente al pago de deudas de las entidades financieras, pues esta mejora de la solvencia al ser comunicada dentro de la información financiera que elaboran estas empresas, repercutirá desde la teoría en un aumento considerable de su transparencia $\mathrm{y}$, en consecuencia, incidirá en el hecho de recuperar la confianza de los inversores.

El necesario papel de la sociedad gestora de activos, según hemos indicado es desarrollado por el Real Decreto-Ley 24/2012 de 31 de agosto, de Reestructuración y Resolución de Entidades de Crédito, complementarias al Real Decreto-Ley 18/2012. Sus medidas van dirigidas a constituir una sociedad de gestión que se encargue de la administración de aquellos activos problemáticos transferidos por las entidades de crédito. En concreto, las entidades que no requieran fondos públicos desarrollarán una actuación temprana, mientras que las entidades que necesiten apoyo financiero y las no viables financieramente, llevarán a cabo actuaciones de reestructuración y resolución, respectivamente, todo ello con la aprobación del Banco de España y bajo la supervisión del FROB. Así, esa necesidad de fondos será cubierta con acciones ordinarias, aportaciones al capital social e instrumentos convertibles en acciones ordinarias. En consecuencia, aumentará la protección del inversor, el poder de la Comisión Nacional del Mercado de Valores y las funciones del Banco de España.

Observamos que en esta sociedad gestora se distinguen dos procesos de actuación, dependiendo de si el tipo de entidad recibe apoyo financiero o no es viable. En ambos casos, los instrumentos emitidos para ayudar a las entidades financieras serán acciones ordinarias, aportaciones al capital social o instrumentos convertibles en acciones ordinarias, sometidos a aprobación del Banco de España y gestionados por accionistas y acreedores. Estos instrumentos podrían favorecer el reparto del capital de la entidad, la entrada de nuevos inversores para mejorar la renovación de la deuda, el aumento de la solvencia de la entidad a medio plazo y, con ello, su valor de mercado y su atractivo para los inversores.

\subsection{Efectos de las principales normativas para las entidades financieras españolas}

Una vez que han sido planteadas todas aquellas normativas más recientes dirigidas al ámbito de las entidades financieras y los principales efectos teóricos perseguidos a través de las mismas, mejora de la solvencia y de la liquidez, pasamos a continuación a analizar algunos de los efectos más relevantes en su aplicación práctica al caso de los bancos españoles. 
En primer lugar destacamos los efectos que ha provocado el Real Decreto-Ley 9/2009 en las entidades financieras. Así, las principales entidades afectadas han sido Catalunya Caixa con una ayuda del FROB de 1.250 millones de euros, Unnim, recibió 280 millones de euros, CEISS, 525 millones de euros, Nova CaixaGalicia, 1.162 millones de euros, BFA Bankia, 4.465 millones de euros, Banco Mare Nostrum, 915 millones de euros y Banca Cívica, 977 millones de euros. En consecuencia, podemos decir que el FROB contribuyó con una ayuda cercana a los 9.500 millones de euros en su intento de consolidar y fortalecer la estructura bancaria del sistema financiero. De esta dotación, la mayor parte de la misma fue destinada a BFA Bankia, mientras que Nova CaixaGalicia y Caixa Catalunya recibieron alrededor de un millón de euros.

Respecto a los efectos provocados por el Real Decreto-Ley 2/2011, debemos mencionar que ante las necesidades de capital de cada entidad, se proponen distintas estrategias de cumplimiento. Así, Grupo Bankia y Grupo Banca Cívica optan como primera opción salir a bolsa, seguida por la ampliación de capital y, por último, la ayuda del FROB. Grupo Mare Nostrum, por su parte, elige como primera opción la ampliación de capital privado, seguida por la salida a bolsa y la generación de capital y, en última instancia, optar a la ayuda del FROB. Grupo "Astur" comparte con Grupo MareNostrum la entrada de capital privado como primera opción, mientras que en segundo lugar opta por la generación interna de resultados y, por último, por la ayuda del FROB. CAM constituye la excepción de todas las entidades afectadas por el Real Decreto-Ley 2/2011, al plantearse la ayuda del FROB la única estrategia. CatalunyaCaixa y Nova CaixaGalicia se plantean recibir la ayuda del FROB junto con la captación de capital privado como única estrategia. Por último, destacamos las entidades que han optado por la integración. Caja España-Duero optó por la integración con Unicaja, mientras que Unnim se plantea la integración con el grupo financiero SIP. Cabe destacar que esta última entidad se plantea como segunda opción la ampliación de capital entre inversores privados y/o el FROB. En este caso, las ayudas recibidas del FROB correspondieron a $1.718 .000 €$ para CatalunyaBank, 568.000 $€$ para Unnim, CEISS, BFABankia, Banco Mare Nostrum y Banca Cívica no recibieron nada mientras que $2.465 .000 €$ recibió Nova CaixaGalicia y Banco de Valencia, $998.000 €$, lo cual supuso un montante global de 5.749.000 €.

Como se puede observar, los efectos de estas normativas se han traducido en importantes ayudas para mejorar la liquidez y solvencia de estas entidades, existiendo claras diferencias en función del grupo en el que se encontraran adscritas. Con lo que podemos deducir desde la práctica la existencia de efectos plausibles en su ejecución.

\section{PAPEL DEL BANCO CENTRAL EUROPEO FRENTE A LA RECESIÓN ECONÓMICA}

A todo lo anterior debemos añadir medidas adicionales tomadas por el Banco Central Europeo para probar la solvencia de los bancos, su capacidad para hacer frente a futuras crisis y examinar la calidad de sus activos. Nos referimos al test europeo de estrés, superado por la banca española al obtener quince de las entidades nacionales sometidas a examen una solvencia de al menos el 5,5 por 100 del capital ${ }^{6}$.

No debemos olvidar la importancia de los Sistemas Institucionales de Protección (SIP) que vinculaban a varias cajas de ahorros y se empezaban a plantear el fin del modelo tradicional de las mismas, al diseñarse uno nuevo que permitía el ejercicio indirecto de la

6 Este ratio lo marca la autoridad como base para hacer frente a una recesión de al menos tres años, según los datos conocidos en octubre de 2010. 
actividad financiera de la caja a través de una entidad bancaria, o la transformación de la misma en una fundación de carácter especial traspasando su negocio a otra entidad de crédito (Larreina, 2013).

Estos SIP también se conocen como fusiones frías, mediante las cuales las entidades que lo conforman mantienen su identidad comercial o crean una sociedad central (un banco) en la que aglutinan los servicios de tesorería, de riesgos y de tecnología. Además, comparten su solvencia y la permanencia de un mínimo de diez años, la liquidez, consolidan sus resultados y acceden con una misma calificación crediticia a los mercados de capitales (Palomo y Gutiérrrez, 2012).

La peculiaridad de los SIP es que permiten a las cajas de ahorros mantener su identidad y personalidad jurídica y no traen consigo aspectos perjudiciales para el ahorrador. Sin embargo, las fusiones frías conllevaron, en última instancia, la intervención de la denominada troika para el rescate de Bankia ${ }^{7}$.

En este entorno tan complicado, el BE solicitó a dos consultoras independientes (Roland Berger y Oliver Wyman) que efectuasen un test de estrés sobre el Sistema Financiero Español, cuyos resultados corroboraron la cantidad de capital a inyectar en aras de rescatar dichas entidades (Wyman, 2012). Es lo que se conoce como rescate bancario bail out o rescate externo a la banca.

Con estas dificultades, el gobierno de España solicitó ayuda financiera de sus socios europeos para el rescate de la banca en junio de 2012. La asistencia financiera europea, fue recogida en un Memorando de Entendimiento en julio de 2012 con el objetivo de reforzar la estabilidad financiera de España (Larreina, 2013). Las condiciones para acceder al mismo fueron un préstamo de hasta 100.000 millones de euros para reforzar la solvencia de las entidades. En este contexto, el gobierno de España aprobó una nueva noma de reestructuración que conllevaba la creación del banco malo o Sociedad de Gestión de Activos procedentes de la Reestructuración Bancaria (SAREB), la cual se creó con el objetivo de la gestión y desinversión ordenada de la cartera de activos recibidos, maximizando su recuperación, en un horizonte temporal de 15 años (Zurita, 2012). Así, la creación de la SAREB permite afrontar la restructuración bancaria de las entidades con problemas (Massó, 2013). Sin embargo, autores como De la Orden (2013) consideran que su denominación no es correcta debido a que su calificación de malo no es positiva y no es un banco sino una sociedad creada con un objetivo muy concreto y respaldada financieramente por el Estado. Incluso, la creación de un banco malo y la recapitalización del sector bancario es condición necesaria para la recuperación económica tan deseada (García y Martín, 2012).

\section{CONCLUSIONES}

A pesar de las distintas medidas legislativas llevadas a cabo por los diferentes países a título individual, así como por la Unión Europea a modo conjunto, los continuos problemas a que se encuentra sometido el sector financiero parecen persistir: liquidez y financiación, deterioro de los activos de las entidades financieras, aumento de los préstamos dudosos, contracción de la demanda, así como el sobredimensionamiento del sistema bancario español, entre otros, lo cual continúa generando cierta desconfianza de los inversores y del público en

\footnotetext{
7 En concreto, la troika está formada por el Banco Central Europeo, el Fondo Monetario Internacional y la Comisión Europea (CE) y estudian la situación económica de los países para señalarles qué medidas y reformas económicas deben llevar a cabo para sanear sus cuentas y crecer. A cambio de obedecer a la troika, el país que lo necesite recibirá financiación del Fondo Monetario Internacional o del Banco Central Europeo y pierde gran parte de su independencia política (Larreina, 2013).
} 
general. Esto nos lleva a plantear si la respuesta regulatoria está produciendo los efectos previstos de estabilidad financiera para el futuro.

La presión regulatoria planteada como respuesta para el sistema financiero y su situación, que ha sido revisada a través de las normativas de mayor impacto y más recientes del caso español por nuestro estudio, nos permite concluir sobre la importancia de tales normativas en la información financiera. En nuestra opinión, el éxito de estas medidas parece estar supeditado a la recuperación de la confianza de los inversores emitiendo nuevas coberturas para los activos inmobiliarios, que permitan paliar las consecuencias económicas de la conocida "burbuja inmobiliaria" en los balances de estas entidades, así como a través del establecimiento de planes de reestructuración para emitir títulos convertibles en acciones o transferir los activos problemáticos a la sociedad gestora de activos y de esta manera incidir, de un modo favorable, en la solución de sus problemas de solvencia.

Lo anterior afecta directamente al papel que la información financiera de estas entidades puede desempeñar como remedio de estos problemas. La existencia de asimetrías informativa complica la situación actual de las entidades financieras y las tareas que puede hacer el gobierno, se mueven entre la exigencia de transparencia informativa, el análisis y la auditoría y la disciplina (White, 2012).

Este estudio ha permitido identificar los dos grandes bloques de actuación que parecen ser tratados por la normativa reciente que afecta a estas entidades, los que afectan a la mejora de la liquidez y aquellos centrados en garantizar la solvencia, los cuales a su vez redundan en una información financiera transparente que refuerce la confianza de ahorradores e inversores. La idea de reducir las asimetrías informativas, no sólo mejorando la situación económicofinanciera de estas entidades, sino apostando por su revelación y comunicación por parte de las mismas, entendemos que desempeña un papel fundamental dentro del proceso de recuperación del sector bancario. Igualmente, nuestro trabajo ha apuntado la necesidad de que la información financiera actúe para la recuperación de la confianza de los inversores, siendo imprescindible la necesidad de comprobar el efecto de tales medidas sobre la evolución experimentada por ciertos indicadores como la medida del riesgo de un país, el Índice de Confianza del Consumidor, el barómetro Edelman Trust Barometer y el indicador de estabilidad financiera, lo cual constituye una futura línea de investigación con la que pretendemos ampliar el presente estudio.

En suma, podemos afirmar que las reformas financieras, siguiendo las condiciones impuestas por los socios europeos, ya se han completado prácticamente. Sin embargo, el coste social y económico de las condiciones asociadas al rescate de la banca española ha sido muy elevado. Que el crédito se siga contrayendo (BE, 2013a y 2013b) y que las entidades de crédito aún no realicen su función tras seis años de crisis, lleva a poner en cuestión el éxito de la reforma financiera.

\section{BIBLIOGRAFÍA}

Alonso Almedia, M. M. y Bueno Campos, E. (2008): "Ética y gobierno de la empresa: base para la confianza de los accionistas”, M@n@gement, 11(2), 211-230.

Aríztegui, J. (2010): El sector bancario español: situación, perspectivas y evolución del proceso de reestructuración, Banco de España, Disponible en http://www.bde.es.

Baliña, S. y Berges, A. (2014): "Flujos financieros y dinámica de la deuda en España", Cuadernos de Información Económica, 240, 27-34.

Banco de España (2011): Resultados de las pruebas de resistencia 2011 para los bancos y las cajas de ahorros españoles, Banco de España, Disponible en http://www.bde.es/. 
- (2012): Proceso de recapitalización y reestructuración bancaria. Resultados de la evaluación independiente del sector bancario español, Banco de España, Disponible en http://www.bde.es/.

- (2013a): Boletín económico mayo, Banco de España, Madrid.

- (2013b): Boletín económico junio, Banco de España, Madrid.

Berges Lobera, A. y Martín Barragán, E. (2011): "Basilea III: un nuevo marco para el negocio bancario y la gestión de riesgos", Boletín de Estudios Económicos, LXVI(202), 5-27.

Bosón Ponte, E., Escobar Rodríguez, T. y Flores Muñoz, F. (2006): “Online transparency of the banking sector", Online Information Review, 30(6), 714-730.

Campa, J. M. y García, J. (2008): "La internacionalización del sector bancario europeo", Universia Business Review, Número especial, 150 Aniversario Banco Santander, Primer trimestre.

Carbó Valverde, S. (2012): "Algunas claves del futuro del sistema bancario", Papeles de Economía Española, 130, 2-20.

Coleman, J. S. (1990): Foundations of Social Theory, Ed. Harvard University Press, Cambridge, Massachusetts.

Clarkson, P. M., Byrnes, W. C., McCormick, K. M., Turcotte, L. P. y White, J. S. (1986): "Muscle soreness and serum creatinine kinase activity following isometric eccentric and concentric exercise", The International Journal of Sports Medicine, 7, 152-155.

Corral Delgado, S., Domínguez Martínez J. M. y López de Paso, R. (2011): "El nuevo mapa del sistema financiero español", Extoikos, 3, 65-77.

Crumpton, M. (2011): “The value of transparency", The Bottom Line: Managing Library Finances, 24(2), 125-128.

Damodoran, A. (2007): "Information transparency and valuation: can you value what you cannot see?", Managerial Finance, 33(11), 877-892.

De La Orden Cruz, M. C. (2013): "La comunicación financiera en tiempos de crisis: el caso de los "bancos malos"”, Historia y comunicación social, 18, 813-822.

Delgado Márquez, B. L., Hurtado Torres, N. E. y Aragón Correa, J. A. (2012): "On the Measurement of Interpersonal Trust Transfer: Proposal of Indexes", Soc Indic Res, 113(1): 433-449.

Doinea, O., Lepadat, G., Tomita, V. y Daniasa, I. (2011): "The role of accounting information in decision-marking strategies and processes", Economics, Management and Financial Markets, 6(2), 188-193.

Dunning, J. H. (1994): Multinational enterprises and the global economy, Edward Elgar Publishing Limited,Wokingham, England.

Ferreira de Mendoza, H., Cordeiro Galvao, D. J. y Falci Villela Loures, R. (2012): "Financial regulation and transparency of information: evidence from banking industry", Journal of Economic Studies, 39(4), 380-397.

Gambetta, D. (1988): Can we Trust Trust, Ed. Trust, Oxford, Londres, Basil Blackwell.

García Mora, A., Hervás Ortega, G. y Romero Paniaga, M. (2007): "El sistema financiero español ante la crisis crediticia internacional”, Ekonomiaz, $63\left(3^{\circ} \mathrm{C}\right), 92-125$.

García Mora, A. y Martín, E. (2012): "Bancos malos: experiencias internacionales y el caso español”, Cuadernos de información económica, 230, 95-103. 
García Soto, M. G. (2003): El gobierno corporativo y las decisiones de crecimiento empresarial: evidencia en las cajas de ahorros españolas, Tesis doctoral, Universidad de las Palmas de Gran Canaria.

Gómez Sala, J. C., Iñíguez Sánchez, R. y Poveda Fuentes, F. (2005): "Revelación voluntaria de información y características de las sociedades cotizadas en el mercado de capitales español”, Revista Española de Financiación y Contabilidad, XXXV, 131, 8-32.

González, A., López, M., Rodríguez, N. y Téllez, S. (2014): "Fiscal policy in a small open economy with oil sector and non-ricardian agents", Desarrollo y Sociedad, 73, 33-70.

Guillén, M. (2004): "La internacionalización de las empresas españolas", Historia Empresarial ICE, 812, 211-224.

Guillén, M. y Tschoegl, A. (2008): "La internacionalización de la banca española”, Universia Business Review, Número especial, 150 Aniversario Banco Santander, primer trimestre 2008.

Hardin, R. (2002): Trust and Trustworthiness, Ed. Russell Sage, New York.

Healy, P. M. y Palepu, K. G. (2001): "Information asymmetry, corporate disclosure and the capital markets: A review of the empirical disclosure literature", Journal of Accounting and Economics, 31(1-3), 405-440.

Josang, A. Lo Presti, S. (2004): Analysing the Relationship Between Risk and Trust. In Dimitrakos, T. (Ed). Proceedings of the Second International Conference on Trust Management, Oxford.

Kang, T. y Pang, Y. H. (2005): "Economic development and the value-relevance of accounting information-a disclosure transparency perspective", Review of Accounting \& Finance, 4 (1), 5-31.

Klotz, L., Horman, M., Bi, H. y Bechtel, J. (2008): "The impact of process mapping on transparency", International Journal of Productivity and Performance Management, 57(8), 623-636.

Larreina Díaz, M. (2013): "La reforma del sistema financiero un año después del rescate: un éxito, pero ¿para quién?”, Boletín de Estudios Económicos, LXVIII(209), 225-262.

Lindqvist, S. (2012): "The concept of transparency in the european union's residential housing market", International Journal of Law in the Built Enviroment, 4 (2), 99-115.

Longinos Marín Rives, J., Arcas Lario, N., Martínez León, I. M, y Olmedo Cifuentes, I. (2012): "Transparencia, gobierno corporativo y participación: claves para la implantación de un código de conducta en empresas de economía social", Revesco, $108,86-112$.

Luhmann, N. (1979): Trust and Power, Ed. Wiley, New York.

Mareque Álvaréz-Santullano, M., López Corrales, F.R. y Pedrosa Rodríguez, M. Á. (2011): La crisis financiera y su incidencia en los informes de auditoría de las entidades cotizadas, En: XVI Congreso de AECA nuevo modelo económico: empresa, mercados y culturas, Granada.

Massó López, G. (2013): "La transformación del sector inmobiliario en España después de la crisis", Boletín de Estudios económicos, LXVIII(209), 293-315.

Muñoz Fernández, G. y Rodríguez Gutiérrez, P. (2011): Responsabilidad Social Corporativa desde el punto de vista de las Entidades Financieras, En XXI Jornadas Hispano-lusas de Gestión Científica, Córdoba. 
Ohmae, K. (1991): El poder de la triada: las nuevas reglas de la competencia mundial, Ed. Mcgraw-Hill, D. L., Madrid.

Ontiveros, E. y Baliña, S. (2012): "La internacionalización de la Economía Española", Mediterráneo Económico, 21, 121-138.

Palomo Zurdo, R. y Gutiérrez Fernández, M. (2012): "El desajuste del crédito en el sistema bancario y la acción de la economía social: el camino de la reestructuración", REVESCO-Revista de Estudios Cooperativos, 109, 138-169.

Parejo Gámir, J. A. (2007). Manual de Sistema Financiero Español. Ed. Ariel, Barcelona.

Pérez, D. y Trucharte, C. (2012): Los ejercicios de estrés test: experiencia reciente y reflexiones sobre su futuro, Banco de España, Disponible en http://www.bde.es.

Popescu, D. (2010): Determinants of Effective Information Transfer in International Regulatory Standards Adoption, Doctoral Thesis, George Washington University.

Putnam, R. (1995): "Bowling alone: America's declining social capital", Journal of Democracy, 6(1), 65-78.

Rodríguez Gutiérrez, P. y Santos Roldán, L. (2011): Responsabilidad social corporativa en entidades financieras: la internacionalización responsable de BBVA y Banco Santander, En: XXI Jornadas Hispano-Lusas de Gestión Científica, Córdoba.

Roth, F. (2011): "The Eurozone crisis and its effects on citizens' trust in the national parliament, FMA Bulletin, 34, 29-30.

Rousseau, D. M., Sitkin, S., Burt, R. y Camerer, C. (1998): "Not so Different After All: A Cross-Disciplinary view of Trust", Academy of Management Review, 23, 393-404.

Sharkie, R. (2009): "Trust in leadership is vital for employee performance", Management Research news, 32(5), 491-498.

Su, H. Y., Shih-Chieh, F. y Chaur Shiuh, Y. (2011): "Relationship transparency for partnership enhancement: an intellectual capital perspective", The Journal of Business \& Industrial Marketing, 26(6), 456-468.

Torrero Mañas, A. (2009): "El futuro del Sistema Financiero Español”, Colección Mediterráneo Económica, 16, 193-202.

White, E. (2012): "Consideraciones acerca del futuro del sector bancario: lecciones tomadas de la historia de la regulación y de la supervisión", Papeles de Economía Española, $130,42-50$.

Wyman, O. (2012): Bank of Spain stress testing exercise, Ed. Oliver Wyman, Madrid.

Zolfalizadeh, M., Farshadfar, S. y Nacheli, E. (2011): "Studying of effective factors on trust level of banks' credit managers with regard to personal characteristics of managers of corporations", Interdisciplinary Journal of Contemporary Research in Business, 2(12), 97-111.

Zurita Ussia, J. (2012): "La reforma del sector bancario español: de la correcta valoración de los activos a la recuperación del crédito", Boletín de Estudios Económicos, LXIX(212), 263-289. 